\title{
NGC 602 Environment, Kinematics and Origins ${ }^{1,2}$
}

\author{
L. Nigra ${ }^{3}$ \\ nigra@astro.wisc.edu \\ J. S. Gallagher, III ${ }^{3}$ \\ jsg@astro.wisc.edu \\ L. J. Smith ${ }^{4,5,6}$ \\ lsmith@stsci.edu \\ S. Stanimirović ${ }^{3}$ \\ sstanimi@astro.wisc.edu \\ A. Nota ${ }^{5}$ \\ nota@stsci.edu \\ and \\ E. Sabbi ${ }^{5}$ \\ sabbi@stsci.edu
}

\begin{abstract}
${ }^{1}$ Based on observations made with the NASA/ESA Hubble Space Telescope, obtained at the Space Telescope Science Institute, which is operated by the Association of Universities for Research in Astronomy, Inc., under NASA contract NAS 5-26555. These observations are associated with program number GO-10396.

${ }^{2}$ Based on observations obtained at the Anglo-Australian Telescope, Siding Spring, Australia.

${ }^{3}$ Department of Astronomy, University of Wisconsin, 475 North Charter Street, Madison, WI 53706-1582, USA

${ }^{4}$ Space Telescope Science Institute, 3700 San Martin Drive, Baltimore, MD 21218, USA

${ }^{5}$ European Space Agency, Research and Scientific Support Department, Baltimore, MD 21218, USA

${ }^{6}$ Department of Physics and Astronomy, University College London, Gower Street, London WC1E 6BT, UK
\end{abstract}


The young star cluster NGC 602 and its associated HII region, N90, formed in a relatively isolated and diffuse environment in the Wing of the Small Magellanic Cloud. Its isolation from other regions of massive star formation and the relatively simple surrounding HI shell structure allows us to constrain the processes that may have led to its formation and to study conditions leading to massive star formation. We use images from Hubble Space Telescope and high resolution echelle spectrographic data from the Anglo-Australian Telescope along with 21$\mathrm{cm}$ neutral hydrogen (HI) spectrum survey data and the shell catalogue derived from it to establish a likely evolutionary scenario leading to the formation of NGC 602. We identify a distinct HI cloud component that is likely the progenitor cloud of the cluster and HII region which probably formed in blister fashion from the cloud's periphery. We also find that the past interaction of HI shells can explain the current location and radial velocity of the nebula. The surrounding Interstellar Medium is diffuse and dust-poor as demonstrated by a low visual optical depth throughout the nebula and an average HI density of the progenitor cloud estimated at $1 \mathrm{~cm}^{-3}$. These conditions suggest that the NGC 602 star formation event was produced by compression and turbulence associated with HI shell interactions. It therefore represents a single star forming event in a low gas density region.

Subject headings: Star Clusters and Associations, Stars, ISM, Galaxies

\section{Introduction}

The young star cluster NGC 602 (J2000 01:29:30, -73:34:0.0) and its associated nebula, N90 are located in the Wing of the Small Magellanic Cloud (SMC). This is the relatively diffuse southeastern region which transitions to the bridge connecting the SMC and the Large Magellanic Cloud (LMC). Massive star formation is naturally a topic of perennial interest and the range of conditions under which stars form at various epochs and host environments makes it a very diverse field. The NGC 602 cluster is an example of massive star formation in a region with diffuse interstellar medium (ISM), having the low metallicity characteristic of the SMC and without any apparent direct kinetic trigger such as a recent nearby supernova. Its isolation alone makes it a great laboratory for the study of star formation since the complexities introduced by kinetic and optical interaction with other star-forming regions are eliminated. This combined with low metallicity and relatively quiescent environment also makes it a candidate for comparison to theoretical work on primordial HII regions driven by Population III stars (e.g., Abel et al. 2007). 
NGC 602 consists of a central star cluster surrounded by the N90 HII region. Its location in the eastern periphery of the SMC is indicated in Figure 1 from the Magellanic Cloud Emission Line Survey (MCELS) 1 . Also shown in the figure is a HI column density image of the NGC 602 neighborhood taken from the HI survey data used in our study described in $\S$ 2. The nominal outline of the $\mathrm{H} \alpha$ supergiant shell SMC-1, identified by Meaburn (1980), is shown and at its southern tip, the nebula $\mathrm{N} 90$ is outlined by a $\mathrm{H} \alpha$ contour. We adopt a distance to the SMC of $60 \mathrm{kpc}$ (Hilditch et al. 2005) and this results in a transverse scale of $0.29 \mathrm{pc} \mathrm{arcsec}^{-1}$.

The $\mathrm{H} \alpha+[\mathrm{NII}]$ Hubble Space Telescope (HST) Advanced Camera for Surveys (ACS) image of NGC 602 and N90 is shown in Figure 2 from which the detailed structure of N90 can be appreciated, but does not do it full justice. A revealing color composite of all three HST ACS images used in this study has been published in Carlson et al. (2007). High angular resolution reveals a very rich structure in the photodissociation regions (PDRs) along ridges and faces to the east, south and west in NGC 602. The nebula is host to many prototypical structures found in HII regions including "elephant trunks" and "pillars of creation" along the northwestern ridge and what is likely a molecular outcropping to the southeast. A catalogue and analysis of the many features found in NGC 602 will be the subject of a new paper.

Also notable are the many galaxies visible both outside and within the nebula, including a prominent grand design spiral seen nearly face-on north of the eastern HII face. The presence of distinct PDRs to the east, south and west, and the openness toward the north suggests a blister morphology. Since the largest dense condensation of gas, which also is in proximity to most of the young stars, is in the south-southeast part of the nebula, it is most likely that the cluster emerged from this region with the first breakout being in the projected northern and western directions. However, even to the south-southeast, there are galaxies visible indicating that there is no obvious remaining extended dense gas concentration there. This transparency suggests a lack of large-scale dust and molecular concentrations anywhere nearby.

The massive stars associated with NGC 602 are in two groups, one large group in the southeast and one smaller group to the northwest. The stellar population of NGC 602 has been studied by Westerlund (1964), Hodge (1983), Hutchings et al. (1991) and most recently by Carlson et al. (2007) and Schmalzl et al. (2008). Westerlund estimated the maximum age at about 10 Myr. Hodge re-analyzed Westerlund's color-magnitude data and placed the age

\footnotetext{
${ }^{1}$ SMC image credit: F. Winkler/Middlebury College, the MCELS Team, and NOAO/AURA/NSF. The image was obtained from the web page http://www.noao.edu/image_gallery/html/im0993.html.
} 
at $16 \pm 5$ Myr. Hutchings et al. obtained optical and UV spectra of the OB stars in the cluster and estimated the age at 5 Myr. Carlson et al., used HST ACS optical and Spitzer IR images and estimated the age of the OB stars at 4 Myr and suggest that the young objects in the two ridges are a result of progressive star formation. Progressive star formation is also suggested by Gouliermis et al. (2007).

The diffuse, quiescent nature of this environment and its low metallicity raises questions as to how massive stars evidently formed in what appears to be a relatively isolated region of dense gas. By analyzing optical images, we look for evidence of the molecular gas substructure left behind in the cluster's formation. In order to understand the kinematic relationship of NGC 602 to the larger scale environment, we compare radial velocities of the HII gas obtained through high resolution $\mathrm{H} \alpha$ longslit spectra to 21-cm HI SMC survey data. We also use the kinematics of HI shells in the region to develop a simple scenario that could have led to the formation of this cluster.

\section{Data}

High resolution images were taken of NGC 602 with the HST ACS (Program GO-10248, PI: A. Nota) using the F555W, F814W and F658N filters (V, I and $\mathrm{H} \alpha+[\mathrm{NII}]$, respectively). A description of these images and their reduction can be found in Carlson et al. (2007). The resolution achieved in these is 0.05 arcsecond FWHM and this results in a spatial resolution of about $0.03 \mathrm{pc}$, or $6300 \mathrm{AU}$.

High spectral resolution observations of the nebula were obtained by Linda J. Smith using the University College London Echelle Spectrograph (UCLES) and the MITLL3 CCD detector at the coudé focus of the $3.9 \mathrm{~m}$ Anglo-Australian Telescope (AAT) on 2006 November 05. A slit of dimensions 1 arcsec $\times 56$ arcsec was used with an interference filter to isolate a single order containing the $\mathrm{H} \alpha$ line. Observations for two slit positions, spanning the central region of the nebula, were obtained at a position angle of $90^{\circ}$. The precise slit positions are shown in Figure 2. The total exposure time for each slit position was 2700 sec. The data were cosmic ray-cleaned, bias-subtracted, and wavelength calibrated. The spectral resolution was measured to be $5.0 \mathrm{~km} \mathrm{sec}^{-1}$ from ThAr arc lines and each spatial pixel is 0.18 arcsec.

The HI structure in the area near NGC 602 was analyzed using a synthesized 21-cm spectral emission map of the SMC described in Stanimirović et al. (1999). The data cube consists of $1.6 \mathrm{~km} \mathrm{sec}^{-1}$ heliocentric velocity slices from 88 to $216 \mathrm{~km} \mathrm{sec}^{-1}$ across the entire SMC with 30 arcsecond spatial resolution and pixel size. A complex set of HI shell 
structures in the SMC has been identified using these data (Staveley-Smith et al. 1997; Stanimirović et al. 1999). The integrated HI column density image derived from this map in the area of the nebula with HI shells overlaid is shown in Figure 3 .

\section{Results}

\subsection{Gas Environment}

The lack of large-scale dense gas and dust in the vicinity of the nebula is established by a systematic search for background objects in the ACS images. The results are shown in Figure 4(a), which shows the locations of detected galaxies throughout the nebula. Background galaxies were located by first visually identifying extended objects in the I-band image, then verifying a corresponding feature in the V-band with no extended feature in the $\mathrm{H} \alpha$ image. Finally, each object was confirmed as non-stellar using SExtractor software (Bertin and Arnouts 1996). Examples of three background galaxies found in the central region are shown in Figure 5 which correspond to locations $\mathrm{A}, \mathrm{B}$ and $\mathrm{C}$ in Figure 4(a). On scales on the order of the nebula, distant galaxies are relatively evenly distributed across the image. The fact that there is a general transparency throughout the nebula indicates that there is no large-scale, dense cloud in the plane of the sky from which this nebula may have formed. In addition, the transparency around the nebula beyond the PDRs indicates that it did not break out from within a large-scale high density molecular cloud complex, but more likely from a small, dense region on the order of the nebula's size or smaller, $\sim 20 \mathrm{pc}$, on the periphery of the southwest cloud.

On smaller scales, there are regions in Figure 4(a) that seem to have a distinct lack of detected galaxies, suggesting an optically thick molecular gas substructure. An example is the region to the south of the apparent molecular outcropping on the southeast face. We would expect to find a clump or filament of molecular gas in this region which would be eroded by the nearby group of OB stars. This apparent substructure is revealed by plotting the density of detected galaxies across the image with higher densities corresponding to higher optical transparency.

First, an otherwise blank image was formed with galaxy location pixels given a value of unity. This galaxy detection map was then convolved with a Gaussian kernel producing a map of relative galaxy density, i.e., transparency, shown in Figure 4(b) with the F555 (V-band) filter image superimposed to provide a reference to the nebular features. The

kernel used had a FWHM of 40 arcsec, indicated by the circle in the image. The scale of the Gaussian kernel was chosen empirically, with smaller scales too sensitive to individual 
galaxies and larger scales suppressing the variation in density. This FWHM corresponds to a physical scale of $12 \mathrm{pc}$ which, appropriately, is comparable to the smallest scale of giant molecular clouds.

Although processing an arbitrary field of galaxies in this manner would also produce an apparent substructure, the dark regions marking obscured zones in Figure 4(b) lie very much where we would expect them to. Most notably, there is a distinct column extending southward from the apparent molecular outcropping on the southeast face toward the region south of the image where there is a high HI column density as shown in the lower image of Figure 1, suggesting a physical connection via dense molecular filaments. There is also evidence of a filament extending from the southwest corner to the north central edge of the image, connecting to the HI region to the south and tracing the northwest PDR and the cluster to the north. We further establish this connection on a kinetic basis as well in $\S$ 3.2.

\subsection{Gas Kinematics}

The maximum HI column density within a $250 \mathrm{pc}$ radius of the nebula is $3.4 \times 10^{21} \mathrm{~cm}^{-2}$. For the SMC HI survey, the column density in the optically thin regime was found to be $N_{H I}<2.5 \times 10^{21} \mathrm{~cm}^{-2}$ (Stanimirović et al. 1999). Since this is only slightly higher than the optically thin limit, we can assume that in this region, we are sampling the greater portion of the neutral hydrogen in the line of sight. There is clearly a relative HI void to the north of the nebula and relatively dense HI to the south. The position of NGC 602 on the edge of the dense HI region suggests that its progenitor molecular cloud may have formed out of the HI cloud structure to the south.

To look for a correspondence, we explored the HI data cube visually by stepping through velocity slices and looking for emission peaks near the nebula. We found a peak of HI to the south-southwest which moved along the lower periphery of the nebula as the central velocity of the slice increased, and diminished when it reached the south-southeast. The effect is that of a clump of HI with a narrow velocity range having a morphology such that it "hugs" the southern half of the nebula. The fact that the velocity sequence traced the lower boundary of the nebula implies a specific connection between the nebula and the cloud. The middle of this HI velocity sequence is $179 \mathrm{~km} \mathrm{sec}^{-1}$ (heliocentric) which is shown in Figure 6. Numbered pixels correspond to velocity profiles sampled around the nebula, some of which are shown in Figure 7. This sequence of plots shows relatively weak components to the west (HI Profile 1) and east (HI Profile 5), with a strong narrow component at the southern periphery (HI Profile 3). The peak velocity increases from west to east as we observed visually. The narrow component in HI Profile 3 has a FWHM of about $6 \mathrm{~km} \mathrm{sec}^{-1}$. 


\subsection{Progenitor Cloud}

Having identified a component of the HI spectrum as a candidate source of progenitor gas for NGC 602, we isolated it by first subtracting a baseline spectral component common to all the pixels in the region. The baseline component spectrum was identified by noting that a group of 5 pixels to the northeast of NGC 602 have a broad baseline spectrum without any discrete components. This baseline spectrum also appears to be a common component to the spectra at all pixels in the vicinity. These pixels are labeled "C" in Figure 6. These baseline HI spectra were averaged and globally subtracted as continuum from the NGC 602 region HI spectrum. The desired cloud component was then defined by making a velocity cut from 170 to $185 \mathrm{~km} \mathrm{sec}^{-1}$.

The integrated column density derived from the resulting cloud component is shown in Figure 8. As expected, there is a distinct clump of enhanced column density south of the nebula which is outlined in the figure. This is NGC 602's progenitor cloud, hereafter referred to as HI J0130-7337+180H2. Partially visible is a filament extending from the northeastern corner of this cloud well away from NGC 602. The HI column density within the boundary shown was integrated and multiplied by an assumed mean atomic mass, $\bar{m}=1.5 m_{H}$, and the cloud was then modeled as a sphere of equivalent mass. There is insufficient data to estimate the spin temperature of the cloud, but Dickey et al. (2000) combined emission and absorption spectra at background source points across the SMC to obtain a sample of cold cloud phase temperatures with a range of about 10 to $150 \mathrm{~K}$. The temperature of HI J0130$7337+180 \mathrm{H}$ is assumed to be in this range. The resulting parameters for the idealized cloud are shown in Table 1 .

\subsection{HI Shell Structure}

Referring to shell structure shown in Figure [3, NGC 602 is located inward of the projected intersection of HI shells 497 and 499, from Staveley-Smith et al. (1997), which are both expanding toward the south-southwest and NGC 602 is situated just inward of the overlap area. Depending on their positions along the line of sight, these two shells could have interacted in the overlap region as they expanded. Given that their centers are near each other on the sky, if they are also in proximity radially, this interaction could have been continuous over a good portion of their history. A super-giant HI shell, SGS 494A from

\footnotetext{
${ }^{2}$ The designation HI J0130-7337+180H describes an HI cloud component at J2000 coordinates $01 \mathrm{~h} 30 \mathrm{~m},-73^{\circ} 37^{\prime}$ with $+180 \mathrm{~km} \mathrm{sec}^{-1}$ heliocentric radial velocity.
} 
Stanimirović et al. (1999), also potentially could interact in recent times with NGC 602 as it expands in the opposite direction from the south-southwest. The parameters of the HI shells in the NGC 602 region are given in Table 2. NGC 602 is in the region where at least three HI shells may interact.

\subsection{HII Region Velocities}

In order to understand the nebula's relationship to the surrounding HI spatial and velocity structure, we derived radial velocities of the ionized gas across the nebula from the AAT UCLES $\mathrm{H} \alpha$ spectroscopy described in $\S$ 2, The mean $\mathrm{H} \alpha$ emission line wavelength was determined by centroiding at each position along the two slits, shown in Figure2, which together span the width of the nebula. Figure 9 is a plot of the mean heliocentric $\mathrm{H} \alpha$ velocity across the nebula derived from these spectra and horizontally scaled in parsecs. The radial velocity covers a range from 174.6 to $184.2 \mathrm{~km} \mathrm{sec}^{-1}$, and the RMS dispersion in radial velocity of the line peak is only $1.4 \mathrm{~km} \mathrm{sec}^{-1}$. The nebula appears to be quite quiescent.

The $\mathrm{H} \alpha$ velocity range across the nebula from the longslit spectra is indicated with a bar above the middle plot in Figure 7. Note that the range is nearly centered with the peak of the narrow southerly HI cloud component and is well-constrained within its range. This strong correspondence between the radial velocity of the ionized gas and position of a prominent HI cloud component in the complex south of the nebula shows that NGC 602 is co-moving with this HI cloud component and therefore likely formed from it.

\section{Discussion}

The part of the Wing of the SMC where NGC 602 resides is characterized by a moderate HI column density of 3 to $4 \times 10^{21} \mathrm{~cm}^{-2}$ and a relatively simple HI shell structure. This total column density is approximately 3 to 4 times the HI star formation threshold for faint dwarf galaxies found by Begum et al. (2006), so in general, star formation might be expected, but is not strongly indicated. The formation of one group of OB stars such as NGC 602 in this environment is therefore not surprising, but the simplicity and diffuse nature of its

surrounding ISM structure, its relative isolation and sparse stellar population lends itself to a straightforward evaluation and comparison of formation scenarios. 


\subsection{Environment}

The low degree of optical obscuration indicated by the presence of galaxies throughout the N90 nebula and its surroundings is consistent with the low levels of extinction in the SMC

Wing reported by Gordon et al. (2003). In that study, the HI column density-to-extinction ratio was sampled at $\mathrm{AzV} 456$, an $\mathrm{OB}$ star $1.6^{\circ}$ to the northwest of NGC 602, and was found to be

$$
N(H I) / A(V)=(7.4 \pm 1.20) \times 10^{21} \mathrm{~cm}^{-2} \mathrm{mag}^{-1} .
$$

The HI column density in the region of the HST image ranges from $1.3 \times 10^{21} \mathrm{~cm}^{-2}$ in the northernmost central point to $3.1 \times 10^{21} \mathrm{~cm}^{-2}$ in the far southwest corner. Using this relationship, the corresponding expected extinction in the visual band (i.e., in the F555W band) ranges from 0.7 to $1.7 \mathrm{mag}$. This is not enough to significantly suppress detection of galaxies, as evident in the HST images. However, as illustrated by Figure 4(b), a locally dense substructure not visible in the lower resolution HI map connects the nebular structures to HI J0130-7337+180H, which is likely a remnant of the progenitor molecular cloud.

\subsection{Kinematics}

Although clearly interacting with its environment, NGC 602 has apparently not produced a significant amount of mechanical energy since it has not significantly disrupted any higher density large-scale HI structures. This is consistent with the young age of the stars which may not have produced any supernovae (see Smith et al. 2008), and the expected low mechanical energy in stellar winds from the OB stars of NGC 602 given the low metallicity of the SMC (Walborn et al. 2000). This lower metallicity leads to reduced stellar wind mass loss rates, which in some cases can be dramatic (e.g., Bouret et al. 2003).

The gentle velocity profile of the ionized gas in Figure 9 supports this picture of relatively peaceful birth and evolution of the gas associated with NGC 602. This cluster is ionizing the diffuse inter-cloud gas and illuminating the surfaces of large-scale structures, while creating smaller-scale structure along the PDRs on cloud peripheries through photoionization, leading to the rich fine structure seen in the HST images (e.g., Figure 2).

\subsection{Origins}

We have established that the morphology and kinematics of the N90 nebula is consistent with cluster formation through a blistering process in the periphery of HI J0130-7337+180H. 
In order to verify that NGC 602's mass is consistent with the cloud's mass, we estimate an upper limit to the star formation efficiency. The total stellar mass has been estimated by Cignoni et al. (2008) at $\sim 2000 M_{\odot}$, corresponding to roughly $\sim 0.7 \%$ star formation efficiency based on the cloud's HI mass estimate given in Table 1. This is only an upper limit because we do not know how much molecular gas exists in the region, but the limit is reasonable. Given that the cluster formed from the cloud, we now consider what could have led to the conditions that allowed core formation and collapse within it.

For the formation of a gravitationally bound molecular region through collapse within the HI cloud, the Jeans length must be smaller than the HI cloud's scale and the the cloud must be undisturbed for a period on order of the free-fall time, $t_{f f}$. Assuming HI J0130$7337+180$ H's precursor was uniform and its global characteristics were similar to those of Table 1 prior to collapse, the Jeans length was less than 15 pc. This is well within the 220 pc scale of the cloud, indicating that bound regions could have formed through simple gravitational collapse. However the time required is $t_{f f} \sim 40 \mathrm{Myr}$, during which time the cloud would have to be undisturbed by significant interactions. This is not likely considering the dynamics of clouds in the SMC. The radial velocity dispersion of shells in the Wing region is $19.4 \mathrm{~km} \mathrm{sec}^{-1}$ (see Table 2), or $27 \mathrm{~km} \mathrm{sec}^{-1}$ transversely on the sky. Although a shell structure is enforced to obtain this value, this would also be the approximate cloud dispersion velocity regardless of the model (i.e., pure turbulence). At this speed, the cloud would need to travel $1.1 \mathrm{kpc}$ or $\sim 1^{\circ}$ on the sky (about half the scale of the SMC Wing) without a significant interaction to experience Jeans instability molecular cloud formation. Since there is insufficient time for spontaneous collapse, some type of interaction is required to accelerate the collapse.

A possible mechanism to stimulate collapse is a gas flow. The HI shell fragments in the region may have been exposed to continuous interactions with their surroundings, encouraging the formation of turbulence. Such a process is described in Slavin et al. (1993) where hot gas flowing past a cool gas cloud can produce an intermediate warm turbulent mixing layer. If, under some conditions, a similar process could lead to further cooling and subsequent star formation in the cloud, it would do so on its periphery. The morphology of the region with NGC 602 forming on the boundary between a cool HI cloud and the large diffuse region to the north seems consistent with this scenario. Interestingly, Hoopes et al. (2002) detected a hot gas component in this sector of the SMC through OVI absorption studies. The velocity spectrum of the hot gas can be interpreted as consistent with a hot outflow or possibly as hot gas flowing through the diffuse HI gas of the Wing.

A perhaps more straightforward mechanism for the required gas interactions are the expanding HI shells discussed in $\S$ 3.4, which would collide and overlap as they expand. 
The radii of shells in a snowplow phase are approximately $R(t)=R\left(t_{0}\right)\left(\frac{t}{t_{0}}\right)^{0.55}$, where the exponent is the average of that for energy- and momentum-conserving modes. The average is used here to simplify approximating the radii. $t_{0}$ is the current dynamical age of the shell as shown in Table 2. In Figure 10 the three shells are shown at the time of their first interaction, at the estimated formation of NGC 602 and at the present time. The earliest interaction occurs at $\sim 6.7$ Myr between shells 497 and 499, assuming their centers are radially at the same position. As they expand, the overlapping region of the shells propagates to the southwest directly toward the current position of the nebula. At 4 Myr, when the NGC 602 cluster began to form, the shells would have been interacting for $\sim 2.7 \mathrm{Myr}$ and may have also had a brief interaction with shell 500. Turbulence, compression and radiative cooling produced in this interaction may have been sufficient to lead to rapid star formation.

A quantitative test of this model would require hydrodynamic simulations of the shell interactions, but potentially relevant 3-dimensional numerical simulations have been performed by Heitsch et al. (2008). Their study showed that uniform, low density warm gas flows in direct collision can lead to instabilities resulting in turbulence and rapid cooling, followed by cloud formation. The initial conditions considered in that study were $n=3 \mathrm{~cm}^{-3}$, $T=1800 \mathrm{~K}$ and $v_{\text {inflow }}=7.9 \mathrm{~km} \mathrm{sec}^{-1}$. Interaction times on the order of 3 Myr were sufficient to cool the warm gas flows in the collision region to a point where $\sim 50 \%$ of the gas was below $100 \mathrm{~K}$ and for dense substructures to form, which plausibly could support star formation.

The initial temperature, density and velocity of the flows in these simulations are on the same order as might be found in the much younger shells at the time they began to interact $\sim 7$ Myr ago. The $3 \mathrm{Myr}$ cooling time found in the simulations is comparable to the estimated 2.7 Myr allowed to establish conditions for star formation in this shell interaction scenario. Although the interaction is not a head-on collision of flows as in the simulations, there are similarities. When two shells begin to interact, the interaction region forms an expanding ring (the intersection of the two spherical shells) which continues to expand until the shells dissipate. The collision region appears locally at any point on the ring as two slabs crossing obliquely and continuously. The obliquely crossing gas ring, therefore, has a head-on velocity component that emulates the simulated interacting flows and also a parallel component which carries the interacting gas with it, expanding the ring.

\section{Conclusions}

The young star cluster NGC 602 and its associated HII region N90 formed in relative isolation in the Wing of the SMC under conditions that are generally marginal for star for- 
mation, the ISM in this region being relatively diffuse and quiescent. A general transparency to galaxies throughout the nebula and its surroundings shows that there are no large-scale dust and molecular concentrations in or near the nebula to explain its formation through simple gravitational collapse. There is also no morphological evidence of violent events such as supernovae or strong stellar winds having directly triggered its initial formation as demonstrated by the low radial gas velocity gradient and dispersion measured across the nebula. Our measured low velocity gradient across the nebula also shows that NGC 602's formation and evolution has also been peaceful and, to our good fortune, has left the evidence of its birth relatively undisturbed. Star formation triggered by gas flow interaction might provide a consistent explanation for NGC 602's formation under these constraints.

Based on a tight, unambiguous neutral and ionized gas velocity correlation at $\sim 180$ $\mathrm{km} \mathrm{sec}{ }^{-1}$, we determined that NGC 602 formed on the periphery of a low-density HI cloud component immediately to its south and blistered from it. The HI density of the natal cloud, designated HI J0130-7337+180H was estimated at $1.3 \mathrm{~cm}^{-3}$ and its size at $220 \mathrm{pc}$ implying a mass of $3 \times 10^{5} M_{\odot}$. This mass is sufficient to provide NGC 602's approximate stellar mass with $\leq 0.7 \%$ star formation efficiency. Given the temperature range of cool cloud components in the SMC of $10 \mathrm{~K}$ to $150 \mathrm{~K}$, the cloud's size is comparable to the Jeans length for a simple gravitational collapse scenario. The timescale for undisturbed collapse is $\sim 40 \mathrm{Myr}$, much longer than the dynamics of the associated HI shells in the SMC would allow. The interaction of large-scale gas components likely accelerated the formation process, producing local molecular concentrations from which the cluster formed. The pattern of transparency to galaxies in the region indicate the presence of fragments of these molecular components in and around the nebula connecting nebular features to the natal cloud.

A simple model of local HI shell evolution shows that two of the expanding shells would have begun interacting $\sim 7 \mathrm{Myr}$ ago and that the nominal path of this interaction region is consistent with the location of NGC 602. Turbulence at the intersection could have led to the formation of this cluster $\sim 3$ Myr later. Further study of the NGC 602 region thus offers an opportunity to explore star formation processes in a low density, relatively quiescent environment.

Support for program G0-10396 was provided by NASA through a grant from the Space Telescope Science Institute, which is operated by the Association of Universities for Research in Astronomy, Inc., under NASA contract NAS 5-26555.

We thank Lynn Carlson for her work in determining the age of the NGC 602 cluster. We thank Fabian Heitsch for his insights into the applicability of colliding flow simulations. We are grateful to the anonymous referee for helping us improve this paper through very 
useful comments. JSG, SS and LN thank the University of Wisconsin Graduate School for their partial support.

\section{REFERENCES}

Abel, T., Wise, J. H., Bryan, G. L. 2007, ApJ, 659L, 87A

Begum, A., Chengalur, J. N., Karachentsev, I. D., Kaisin, S. S., \& Sharina, M. E. 2006, MNRAS, 365, 1220

Bertin, E., \& Arnouts, S. 1996, A\&AS, 117, 393

Bouret, J. C., Lanz, T., Hillier, D. J., Heap, S. R., Hubeny, I., Lennon, D. J., Smith, L. J., Evans, C. J., 2003, ApJ, 595, 1182

Carlson, L. R., Sabbi, E., Sirianni, M., Hora, Nota, A., Meixner, M., Gallager, J. S., III, Oey, M. S., Pasquali, A., Smith, L. J., Tosi, M., Walterbos, R. 2007, ApJ, 665, L109

Cignoni, M. et al. 2008, in preparation.

Dickey, J. M., Mebold, U., Stanimirovic, S., \& Staveley-Smith, L. 2000, ApJ, 536, 756

Gordon, K. D., Clayton, G. C., Misselt, K. A., Landolt, A. U., \& Wolff, M. J. 2003, ApJ, 594,279

Gouliermis, D. A., Quanz, S. P., Henning, T. 2007, ApJ, 665, 306

Heitsch, F., Hartmann, L. W., Slyz, A. D., Devriendt, J. E. G., \& Burkert, A. 2008, ApJ, 674,316

Hilditch, R. W. , Howarth, I. D. \& Harries, T. J. 2005, MNRAS, 357, 304

Hodge, P. W. 1983, ApJ, 264, 470

Hoopes, C. G., Sembach, K. R., Howk, J. C., Savage, B. D., \& Fullerton, A. W. 2002, ApJ, 569,233

Hutchings, J. B., Cartledge, S., Pazder, J., \& Thompson, I. B. 1991, AJ, 101, 933

Meaburn, J. 1980, MNRAS, 192, 365

Slavin, J. D., Shull, J. M. \& Begelman, M. C., 1993, ApJ, 407, 83 
Schmalzl, M., Gouliermis, D. A., Dolphin, A. E., Henning, T., 2008, ArXiv e-prints, 0804, 0543

Smith, L. J. 2008, in preparation.

Stanimirović, S., Staveley-Smith, L., Dickey, J. M., Sault, R. J., \& Snowden, S. L. 1999, MNRAS, 302, 417

Staveley-Smith, L., Sault, R. J., Hatzidimitriou, D., Kesteven, M. J., \& McConnell, D. 1997, MNRAS, 289, 225

Walborn, N. R., Lennon, D. J., Heap, S. R., Lindler, D. J., Smith, L. J., Evans, C. J., \& Parker, J. W. 2000, PASP, 112, 1243

Westerlund, B. 1964, MNRAS, 127, 149 
Table 1. Parameters for SMC cloud HI J0130-7337+180H ${ }^{a}$

\begin{tabular}{|c|c|c|}
\hline Diameter & $\mathrm{d}$ & $220 \mathrm{pc}$ \\
\hline HI density & $n_{H I}$ & $1.3 \mathrm{~cm}^{-3}$ \\
\hline Mass density & $\bar{\rho}$ & $3.2 \times 10^{-24} \mathrm{gm} \times \mathrm{cm}^{-4}$ \\
\hline Mass & M & $2.7 \times 10^{5} \mathrm{M}_{\odot}$ \\
\hline Spin Temperature & $\mathrm{T}$ & 10-150 K \\
\hline
\end{tabular}

a Modeled as a uniform sphere. 
Table 2. Characteristics of HI shells near NGC $602^{\mathrm{a}}$

\begin{tabular}{|c|c|c|c|c|c|c|}
\hline Shell ID & $\begin{array}{c}\text { Center Distance } \\
\text { from NGC } 602^{\mathrm{b}} \\
(\mathrm{pc})\end{array}$ & $\begin{array}{c}\text { Center Angle } \\
\text { wrt NGC } 602^{\mathrm{b}} \\
\left({ }^{\circ} \mathrm{E} \text { of } \mathrm{N}\right)\end{array}$ & $\begin{array}{c}\text { Radius }^{\mathrm{c}} \\
(\mathrm{pc})\end{array}$ & $\begin{array}{c}\text { Heliocentric } \\
\text { Velocity } \\
\left(\mathrm{km} \times \mathrm{sec}^{-1}\right)\end{array}$ & $\begin{array}{c}\text { Expansion } \\
\text { Velocity } \\
\left(\mathrm{km} \times \mathrm{sec}^{-1}\right)\end{array}$ & $\begin{array}{c}\text { Dynamical } \\
\text { Age } \\
\text { (Myr) }\end{array}$ \\
\hline SGS 494A & 670 & 193.3 & $650 / 540$ & 159 & 28 & 12 \\
\hline 497 & 150 & 341.0 & 194 & 180.3 & 13.4 & 8.5 \\
\hline 499 & 130 & 32.3 & 179 & 160.7 & 14.2 & 7.4 \\
\hline 500 & 90 & 121.6 & 233 & 163.0 & 15.4 & 8.8 \\
\hline
\end{tabular}

${ }^{a}$ From Staveley-Smith et al. (1997) and Stanimirović et al. (1999).

${ }^{\mathrm{b}}$ NGC 602 position is RA 1:29:30, DEC -73:34:00 (J2000).

${ }^{\mathrm{c}}$ Radius for spherical models, semi-major/semi-minor axes for ellipsoidal models. 


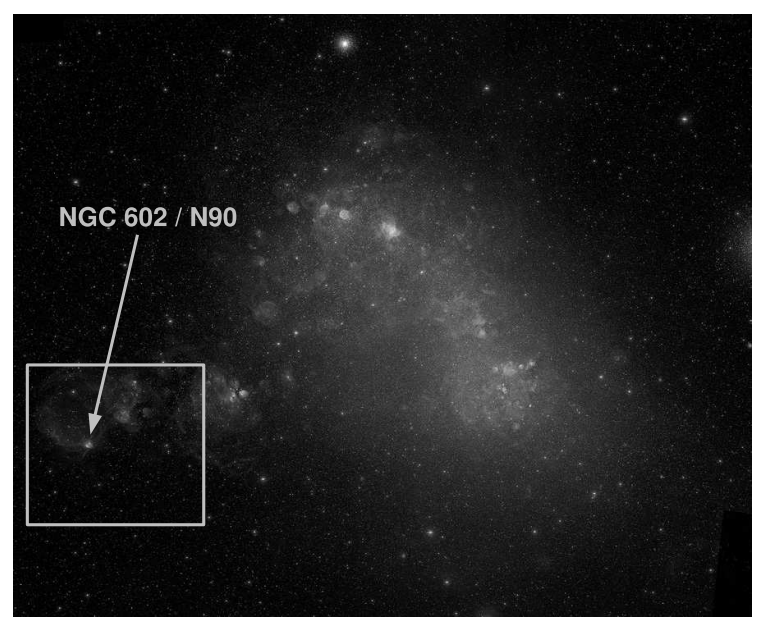

(a) NGC 602 in the Wing of the SMC

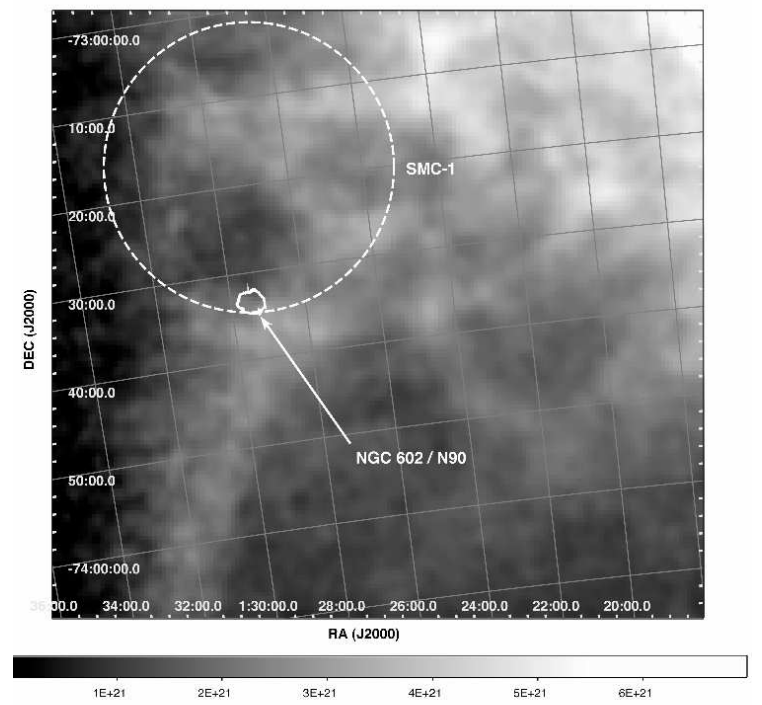

(b) NGC $602 \mathrm{HI}$ environment

Fig. 1.- (a) NGC 602 and its associated HII region, N90 is located in the southeastern corner in the Wing of the SMC shown here as an emission line composite in the upper frame (Composite image credit: F. Winkler/Middlebury College, the MCELS Team, and NOAO/AURA/NSF). (b) The region around NGC 602 indicated in the upper frame is expanded and shown in the lower frame as a HI column density image taken from the data developed by Stanimirović et al. (1999). The H $\alpha$ supergiant shell, SMC-1 (faintly visible in the upper frame) is roughly outlined on the HI image and the nebula, N90 is outlined at SMC-1's southern tip by a deep H $\alpha$ contour taken from the F658N HST ACS image. The intensity scale is in units of $\mathrm{cm}^{-2}$. 


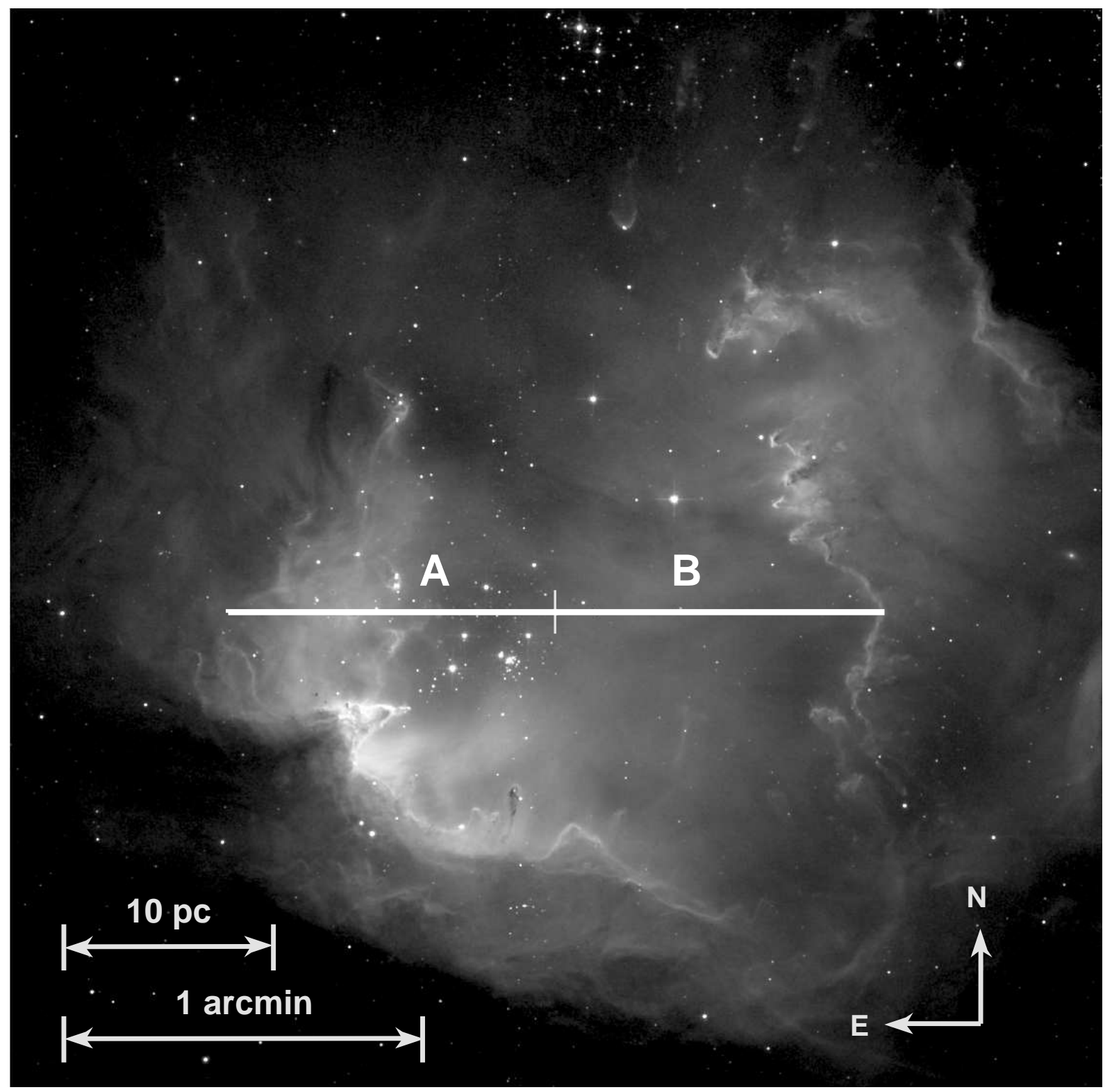

Fig. 2.- NGC 602/N90 H $\alpha$ image from the HST ACS reveals a rich PDR structure with many "elephant trunks" and prototypical HII region features. Overlaid on the image are the two slits used with the AAT UCLES to obtain the HII velocity profile across the nebula shown in Figure 9. 


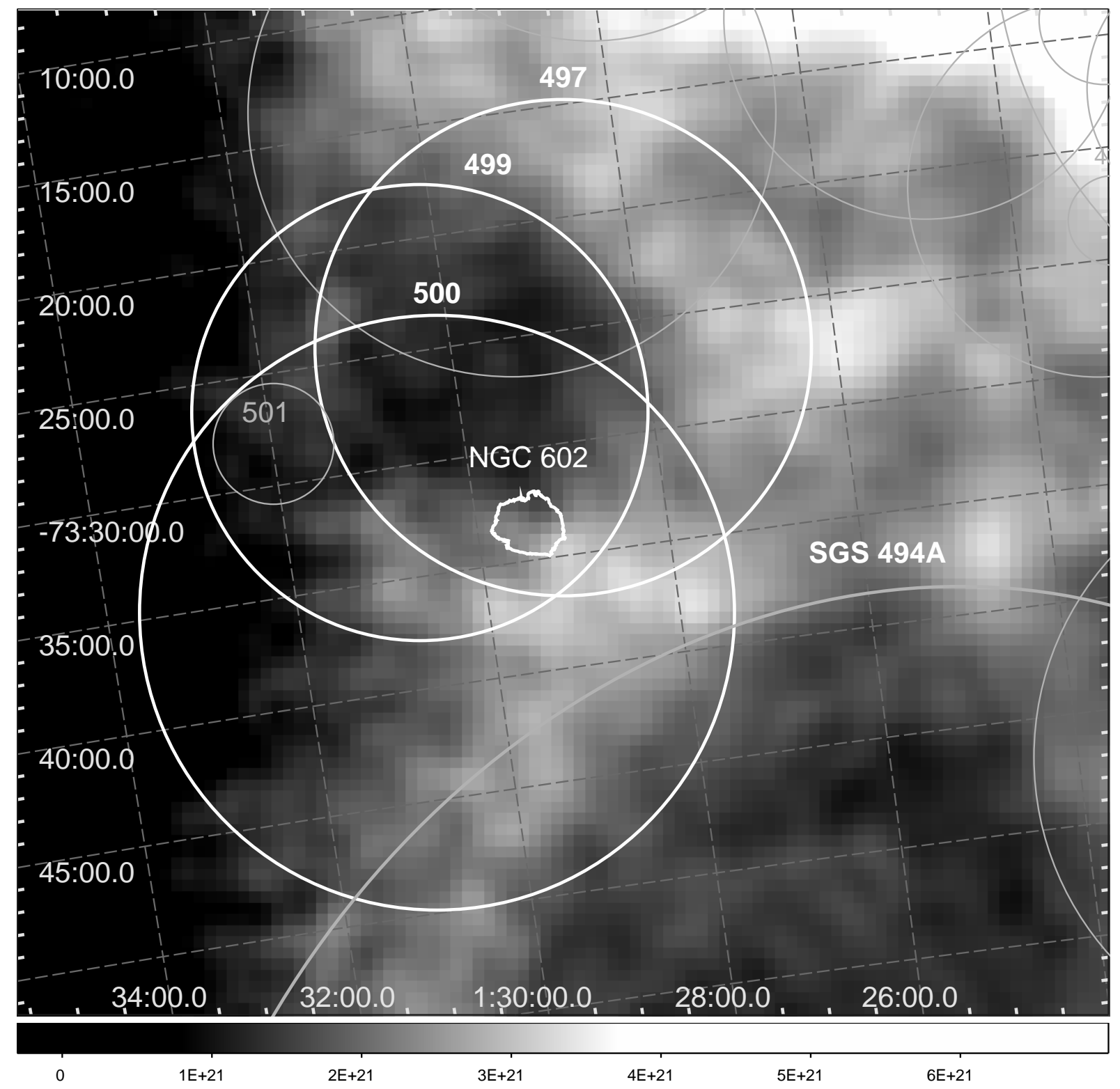

Fig. 3.- HI total column density image for the vicinity of NGC 602 , shown as an outline of its $\mathrm{H} \alpha$ emission region. HI shells identified by Staveley-Smith et al. (1997) and Stanimirović et al. (1999) are overlaid with shells 497, 499 and 500 highlighted. These shells are situated such that they are candidates for influencing the formation of NGC 602. Super giant shell SGS 494A may have influenced the present structure. Coordinates are J2000. The intensity scale is in units of $\mathrm{cm}^{-2}$. 


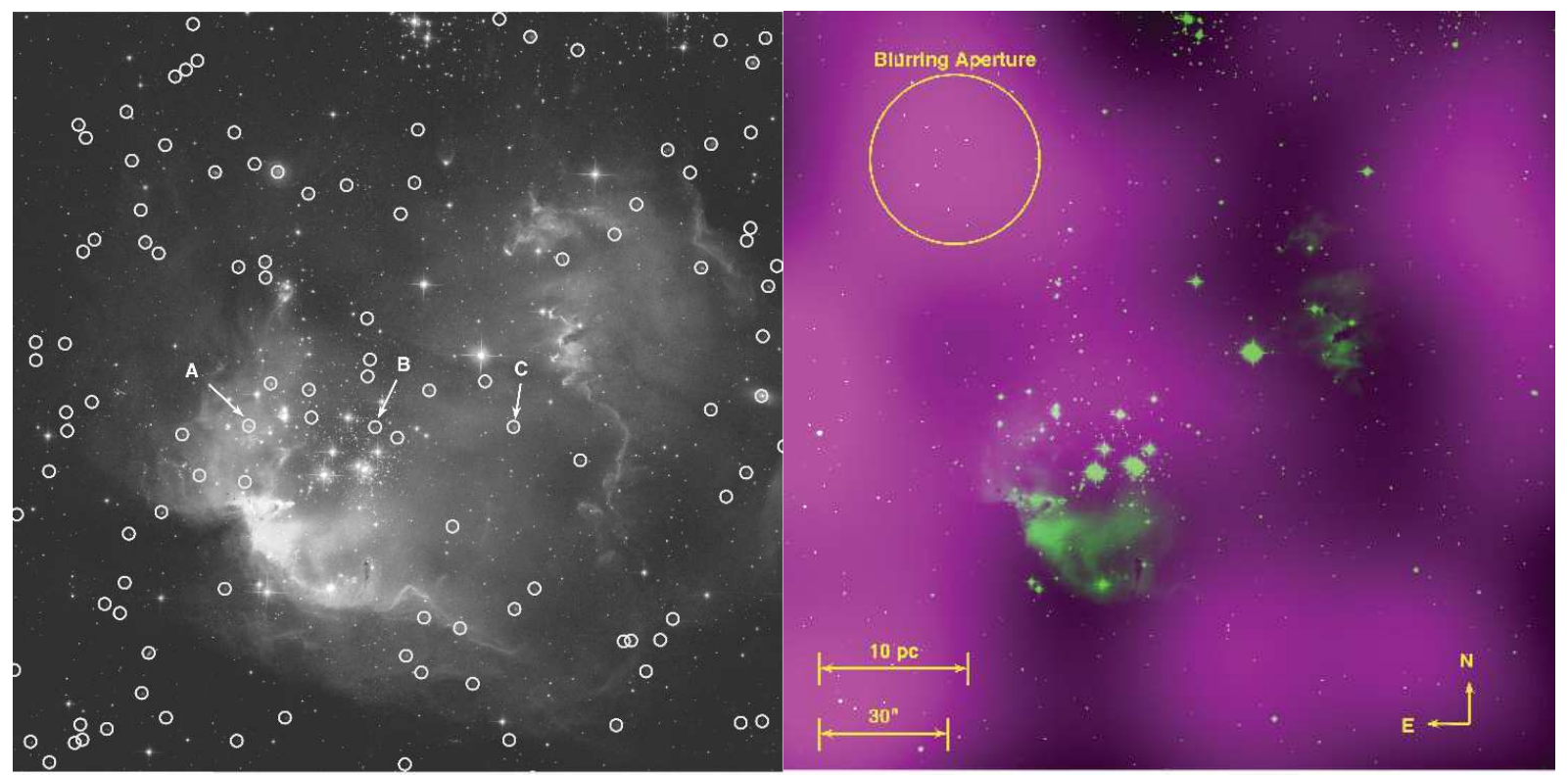

(a) Galaxy locations

(b) Transparency map

Fig. 4.- (a) Background objects visible in the NGC 602 region are identified by circles overlaid on a $\mathrm{V}+\mathrm{I}+\mathrm{H} \alpha$ composite image. The objects were identified visually in the I-band image, required a corresponding feature in $\mathrm{V}$ but none in $\mathrm{H} \alpha$ and finally, confirmed as a non-stellar object by SExtractor in I-band. The three sample objects identified as A, B and $\mathrm{C}$ are expanded and shown in Figure 5, (b) A transparency map created by mapping the density of detected galaxies. The density map was obtained by convolving a map of detected galaxy locations (each a single pixel with value of unity) with a Gaussian kernel whose FWHM aperture is shown in the figure. This image is shown in magenta (brighter is more transparent) while the V-band ACS image is overlaid in green for reference. The image intensity scale is arbitrary. 


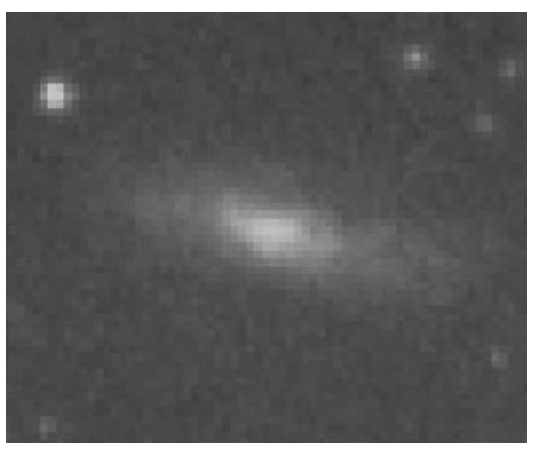

(a) Object A

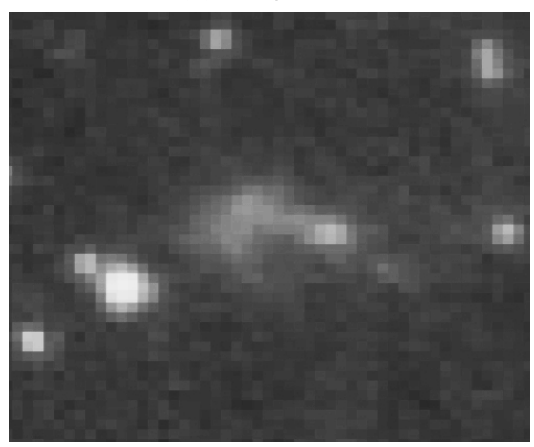

(b) Object B

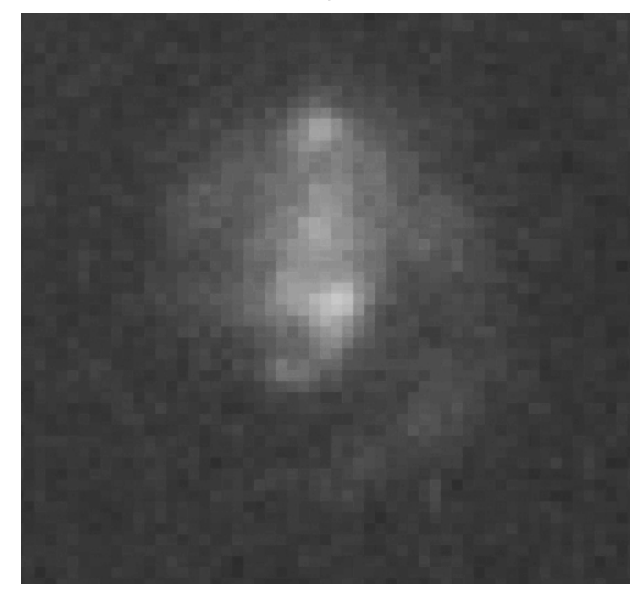

(c) Object C

Fig. 5.- Example background galaxies expanded in the central region shown in I band only. Letters A, B and C correspond to locations identified in Figure 4. 


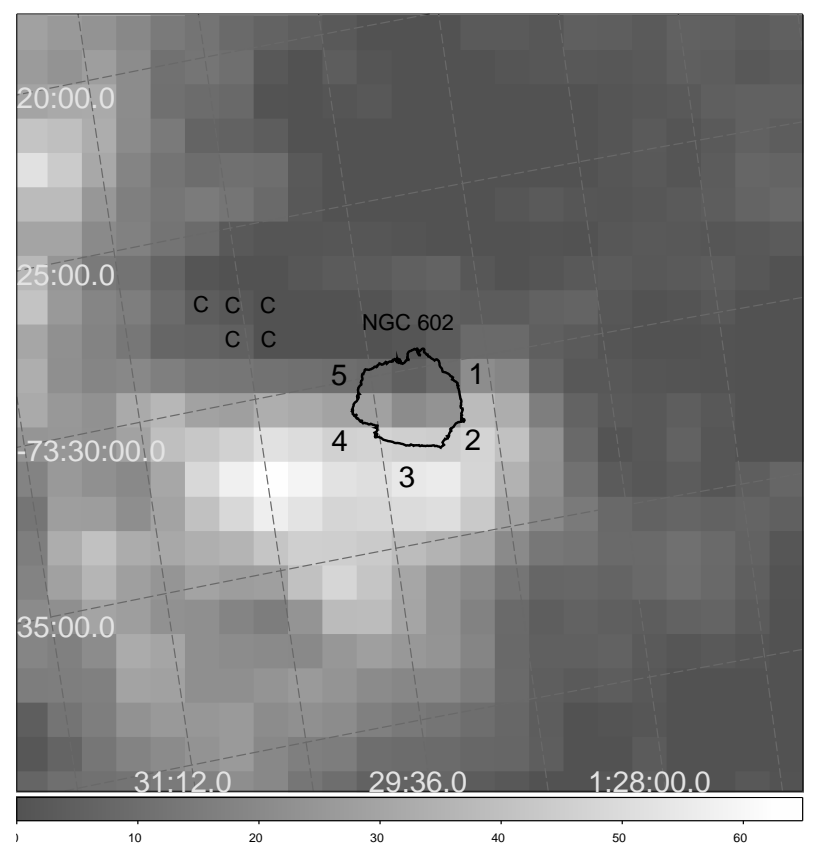

Fig. 6. - The data cube slice at $179.2 \mathrm{~km} \mathrm{sec}^{-1}$ heliocentric velocity where HI intensity peaks south of the NGC 602 HII region (outlined) and correlates with the HII velocity across the nebula. The numbered pixels correspond to velocity profile samples around the nebula. The pixels labeled with "C" have been identified as having a profile representative of a baseline common to all pixels in the area. Coordinates are J2000. The intensity scale is brightness temperature in $\mathrm{K}$ within the $1.6 \mathrm{~km} \mathrm{sec}^{-1}$ channel. 

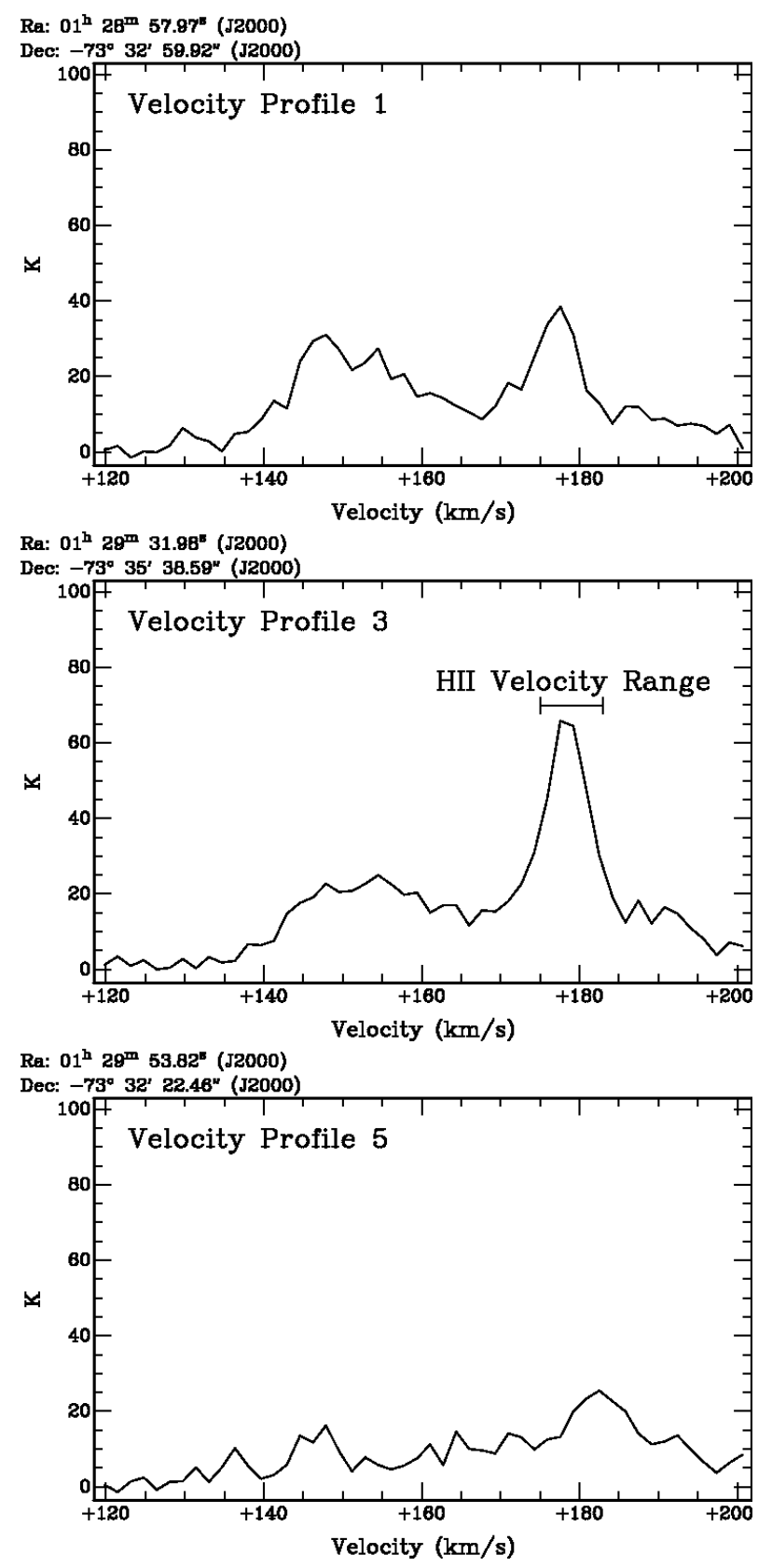

Fig. 7.- HI velocity profiles sampled around NGC 602. Location numbers correspond to those in Figure 6. The HII velocity dispersion found across the nebula is indicated by the bar above the component in profile 3 . 


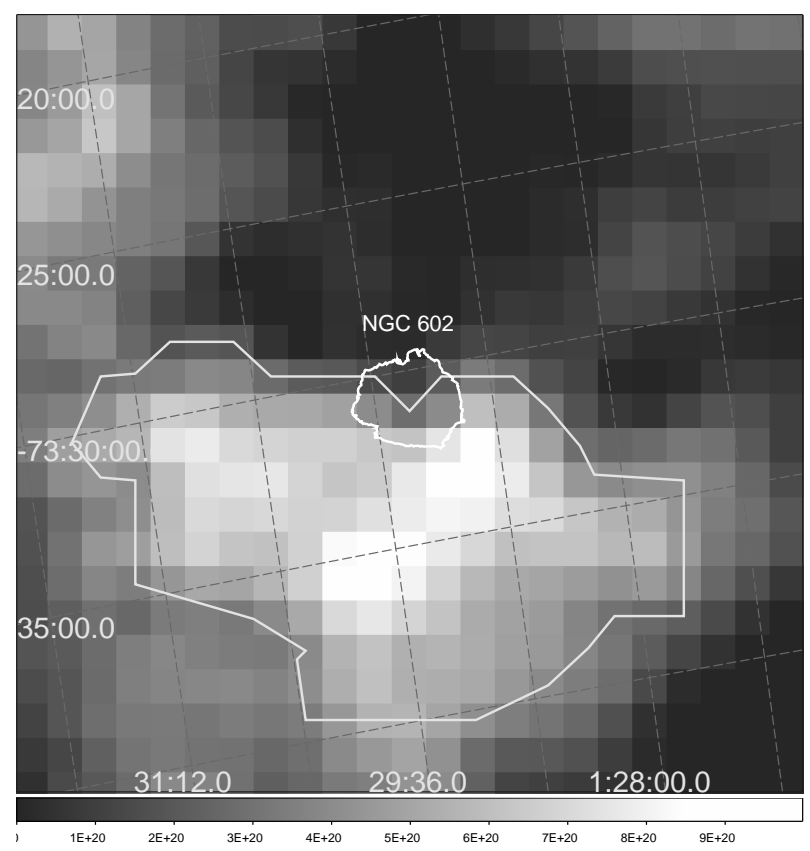

Fig. 8.- Boundary of isolated cloud component HI J0130-7337+180H used in progenitor cloud calculations. The image represents the integrated column density after removing the baseline continuum component and making a velocity cut from 170 to $185 \mathrm{~km} \mathrm{sec}{ }^{-1}$. Coordinates are J2000. The intensity scale is in units of $\mathrm{cm}^{-2}$.

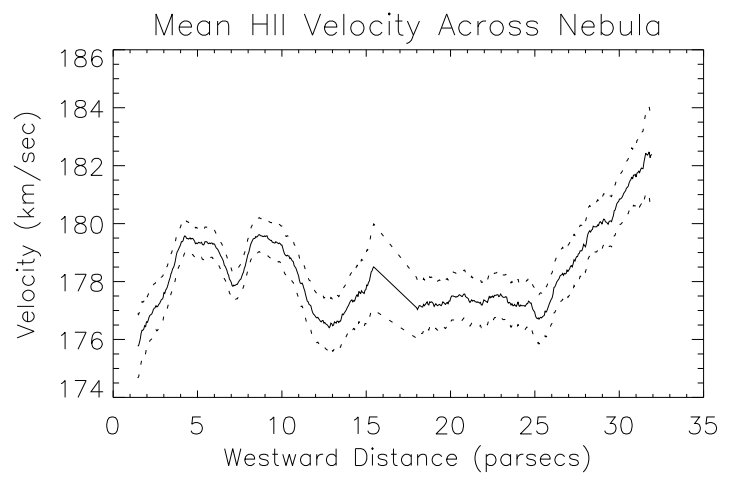

Fig. 9.- The mean HII velocity derived from the two slits, (solid line) smoothed to $\sim 1 \mathrm{pc}$ with approximate position along the nebula from east to west as the horizontal axis. Distorted data due to edge effects on the spectra and from smoothing is suppressed. Linear interpolation was used to fill the resulting gap in the middle of the plot. The dashed lines represent the centroiding error limits $(1 \sigma)$. Including error, the minimum and maximum velocities are 174.7 and $184.1 \mathrm{~km} \mathrm{sec}^{-1}$, respectively. 


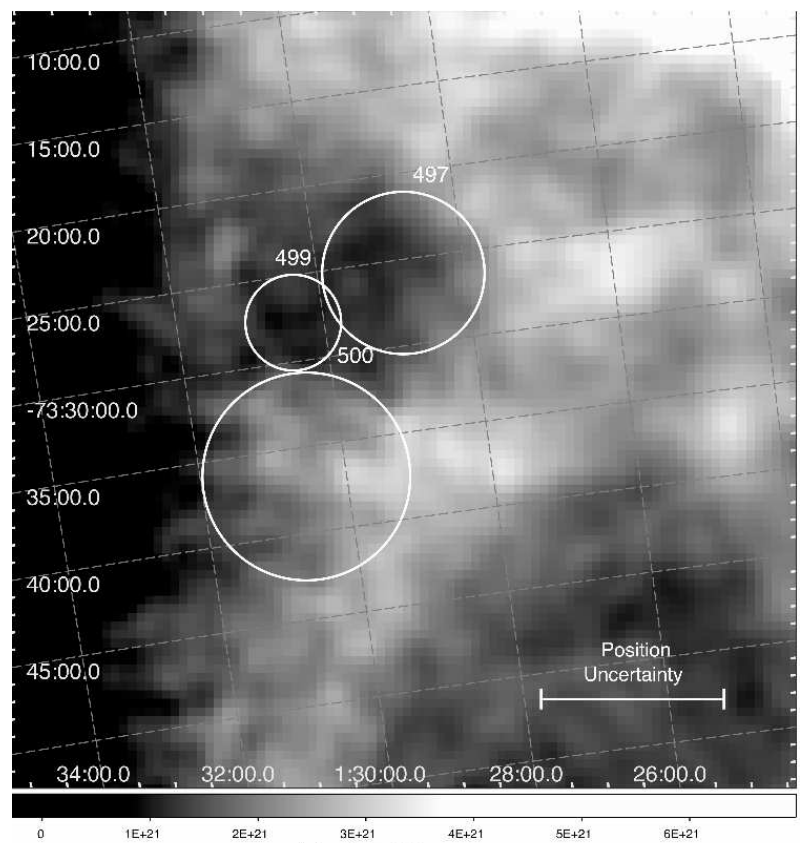

(a) $6.7 \mathrm{Myr}$ ago

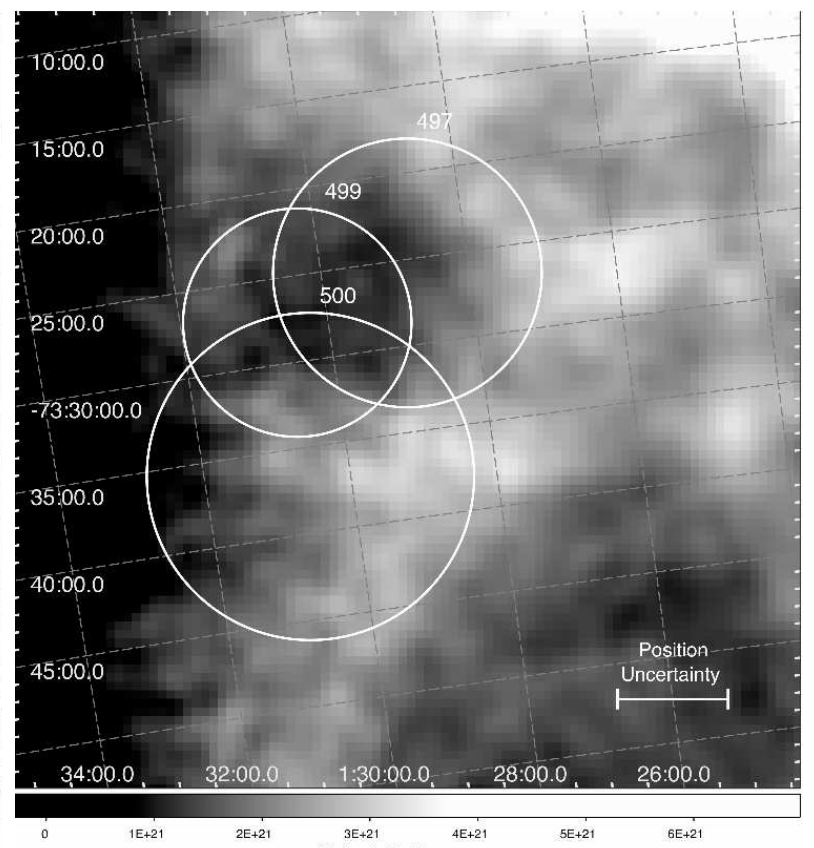

(b) $4 \mathrm{Myr}$ ago

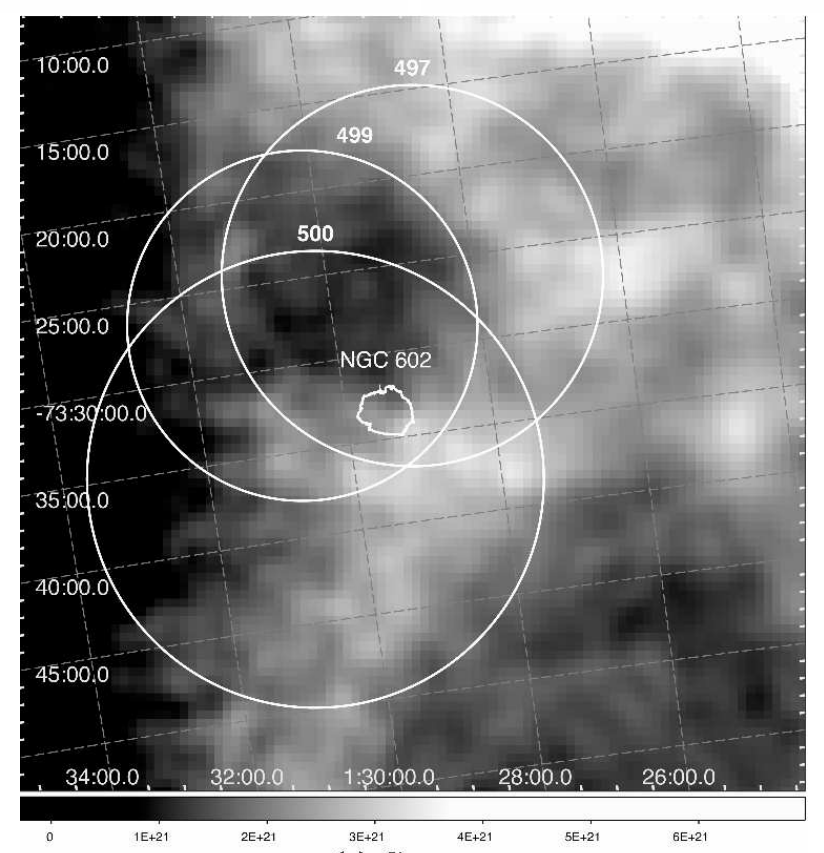

(c) Current

Fig. 10.- Estimated shell evolution and possible interaction leading to NGC 602 formation. The estimated transverse shell position uncertainty is indicated by the bar in each diagram. This is calculated as $\sigma_{t}=\sqrt{2} \sigma_{v r} t$ where $\sigma_{v r}=19.4 \mathrm{~km} \mathrm{sec}^{-1}$ is the radial velocity dispersion in the SMC Wing region (Staveley-Smith et al. 1997). Coordinates are J2000. The intensity scale is in units of $\mathrm{cm}^{-2}$. 